\title{
Effect of intravenous iron dextran on rheumatoid synovitis
}

\author{
D R BLAKE, ${ }^{1} \mathrm{~J}$ LUNEC, ${ }^{1}$ M AHERN, ${ }^{2}$ E F J RING, ${ }^{2}$ J BRADFIELD ${ }^{3}$ AND \\ J M C GUTTERIDGE
}

From the ${ }^{1}$ Rheumatism Research Wing, the Medical School, University of Birmingham, Birmingham B15 2TJ; the ${ }^{2}$ Royal National Hospital for Rheumatic Diseases, Upper Borough Walls, Bath BA1 1RL; the ${ }^{3}$ Dept of Pathology, University of Bristol, Bristol BS2 8HW; and the ${ }^{4}$ National Institute of Biological Standards and Control, Hampstead, London NW3 6RB

SUMMARY Eleven patients with rheumatoid arthritis received a total dose infusion of iron dextran for anaemia. Two of them had anaphylactic reactions and the remaining nine an exacerbation of synovitis. Quantitative infrared thermal imaging was used to assess the extent and distribution of joint involvement resulting from this therapy. In all eight patients examined the 'thermographic index' increased in two or more joint areas, indicating an increase in inflammation. Small joints of the hands were maximally affected, though larger joints when previously inflamed also worsened. Uninflamed joints were rarely affected. The exacerbation of synovitis occurred $24-48 \mathrm{~h}$ after completion of the iron dextran infusion and corresponded with a saturating of the serum iron binding capacity. Levels of immune complexes were unaltered, implying normal reticuloendothelial function. In one further patient, reported to have synovial flares when challenged with oral ferrous sulphate, iron dextran was infused at a lower dosage. All previously inflamed joints in this patient worsened $12 \mathrm{~h}$ after the infusion was discontinued. Concomitant with this was an increase of lipid peroxidation products in synovial fluid and to a less extent serum. Iron dextran in vitro stimulated lipid peroxidation, but dextran alone had no effect. It is therefore suggested that iron dextran worsens synovial inflammation by promoting lipid peroxidation.

Key words: inflammation, lipid peroxidation, reticuloendothelial blockade, immune complexes, anaemia.

Total dose infusions of iron dextran have been reported to exacerbate symptoms of rheumatoid arthritis (RA). ${ }^{1-4}$ The incidence of this reaction is reported to vary. Between 5 and $100 \%$ of patients receiving iron dextran are affected, and the mechanism of toxicity is still undefined. Suggestions so far proposed include: (1) a hypersensitivity to the dextran; (2) a direct toxic effect of iron within the synovial membrane, possibly by the generation of toxic free radicals and lipid peroxides; ${ }^{2}$ and (3) a block in reticuloendothelial cell (REC) function resulting in a secondary immune complex synovitis. ${ }^{6}$

Here we have studied 11 patients with rheumatoid

Accepted for publication 20 September 1984.

Correspondence to Dr D R Blake, Rheumatism Research Wing, Medical School, University of Birmingham, Birmingham B15 2TJ. arthritis who had been prescribed IV iron dextran by their physician for the treatment of 'rheumatoid anaemia'. The object of the study was to assess: (1) the incidence and features of the synovial reaction; (2) whether the mechanism is indeed an immune complex synovitis, secondary to REC blockade; or (3) whether it relates to iron toxicity mediated by the process of lipid peroxidation.

\section{Patients and methods}

All patients fulfilled the criteria laid down by the American Rheumatism Association for classical or definite rheumatoid disease, ${ }^{7}$ and developed arthritis as adults. One patient, described separately, developed her arthritis as a child (see below, 'Case 
history). Iron dextran (Imferon) was infused in $\mathbf{5 0 0}$ $\mathrm{ml}$ of $5 \% \mathrm{w} / \mathrm{v}$ dextrose saline or $0.15 \mathrm{M} \mathrm{NaCl}$ over an 8-12 $\mathrm{h}$ period according to the manufacturers' recommendation (dose - as in 'Results'). Hydrocortisone was added to the infusion in one case only ('Case history'). All patients were confined to bed for four days, being ambulant for thermographic studies only, and hospitalised until the end of the assessments.

Quantitative infrared thermal imaging was used to assess serially the distribution and extent of the synovial reaction. The skin temperature over six separate joint areas (hands and wrists, knees, ankles) was assessed from isothermograms by a standardised technique described previously. ${ }^{8}$ By analysis of the isotherm areas a thermographic index is calculated from a region of interest defined for each joint image. The index is an objective measure of joint temperature after cooling in a controlled ambient at $20^{\circ} \mathrm{C}$. Assessments were made prior to the infusion, then on four occasions in the following seven days, and intermittently until the reaction had settled. All assessments were made between the hours of 9 am and 11 am to overcome known diurnal variations in joint temperature. ${ }^{9}$

Laboratory measurements performed before and during the study period included a measurement of haemoglobin with red cell indices, and serum ferritin (Gammadab ${ }^{125}$ I ferritin kit, Travenol Laboratories), serum iron, and iron binding capac- ity. Immune complexes were measured by three? functionally separate assays as described by Blake $e \vec{F}$. $a{ }^{9}$ and included platelet aggregation, ${ }^{125} \mathrm{IC} 1 \mathrm{q}^{5}$ binding, and anticomplementary activity. Complement components $\mathrm{C} 3$ and $\mathrm{C} 4$, were measured by radial immunodiffusion. REC function was also investigated in mice, with hepatic and splenic uptake of chromium-labelled sheep red blood cells adminisen tered intravenously. Details of the technique are published elsewhere. ${ }^{10}$ In this animal study irondextran (20 mg in $0.4 \mathrm{ml} 0.15 \mathrm{M} \mathrm{NaC1}$ ) wasw administered to six mice intravenously via the latera tail vein and compared with dextran sulphate, molo wt $900000(0.5 \mathrm{mg}$ in $0.2 \mathrm{ml} 0.15 \mathrm{M} \mathrm{NaC} 1)$ and carbon suspension (16 mg in $0.2 \mathrm{ml} 0.15 \mathrm{M} \mathrm{NaC} 1)$ ași positive controls, and with $0.15 \mathrm{M} \mathrm{NaC1}(0.2 \mathrm{ml})$ as. a negative control.

In one patient only the products of lipid peroxida 9 tion were measured serially in fresh sera and synovial fluid, as stored $\left(-20^{\circ} \mathrm{C}\right)$ sera provectiv unsatisfactory. Thiobarbituric acid (TBA) reactives material and diene conjugates (DC) were analysed by the methods of Satoh ${ }^{11}$ and Lunec anco Dormandy ${ }^{12}$ respectively.

Fig. 1 illustrates the place in the autocatalytic sequence of peroxidation of polyunsaturated fattys acids (PUFA-lipid peroxidation) at which DC anक TBA reactive materials are formed. Initiation of lipid peroxidation in a.lipid membrane is due to the attack of any species that has sufficient reactivity to

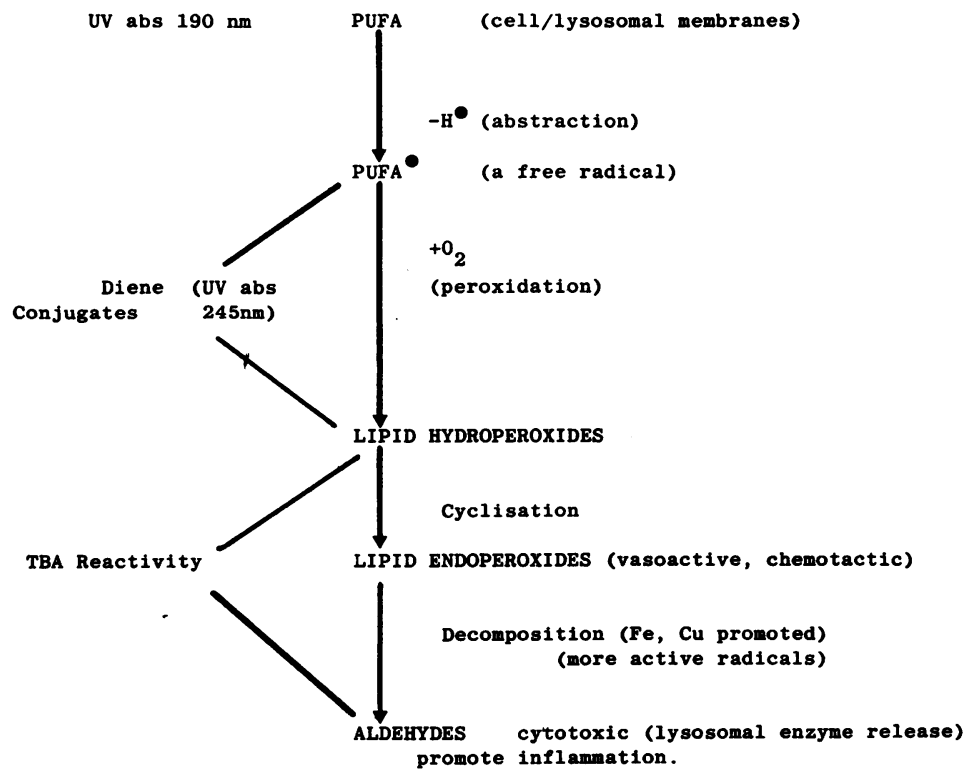

Fig. 1 Scheme illustrating the proinflammatory activity and variety of products generated by the peroxidation of polyunsaturated fatty acids (PUFA). 
abstract a hydrogen atom, thereby leaving an unpaired electron on the carbon atom (carbon radical). This is stabilised by a molecular rearrangement producing a conjugated diene, which then reacts with $\mathrm{O}_{2}$ to give a lipid hydroperoxide. The former can be measured directly, by its absorbance at $245 \mathrm{~nm}$ or indirectly (together with further degradation products) by its ability to form a chromogen with TBA (for a review see McBrien and Slater ${ }^{13}$ ).

\section{Results}

In 10 patients $1 \mathrm{~g}$ intravenous iron dextran infusion was commenced without hydrocortisone cover. Two of these patients developed an anaphylactic reaction consisting of an abrupt (over a few seconds) sensation of malaise, followed by dyspnoea and wheezing. In both cases a profound tachycardia was noted, and the blood pressure was transiently unrecordable. Both responded promptly to $200 \mathrm{mg}$ of intravenous hydrocortisone and the cessation of iron therapy. The reaction developed 20 and 75 minutes into the infusion and after the 'slow infusion' period recommended by the manufacturers.

The eight patients who completed a total dose infusion of $1 \mathrm{~g}$ all showed an increased inflammation as quantified and assessed by infrared thermal imaging. These detrimental changes could be detected by clinical observation in four of the patients. Fig. 2 details the changes observed in patient 1 by plotting thermographic index against time. Data of all eight patients are shown in Table 1 . Small joints of the hands and wrists were maximally affected, though other previously inflamed joints worsened to some extent in almost all patients. Previously uninflamed joints rarely showed an inflammatory reaction. The onset of the inflammatory reaction was between the first and third day and settled in all patients within 11 days. The extent, or onset, of the rise showed no relationship to the initial serum ferritin value and preceded the maximum rise in serum ferritin (days 3-9), and further was not related to the extent of the rise. In three patients daily assessments of serum iron and iron binding capacity were performed. The iron binding capacity remained fully saturated until the fourth day, when usually the reaction began to settle.

Immune complex levels (assessed by any one of the three assays) did not change significantly during the reaction, and complement levels $\mathrm{C} 3$ and $\mathrm{C} 4$ were unaltered. In mice clearance of hepatic and splenic chromium-labelled sheep red blood cells, following iron dextran treatment, was not appreciably different from the saline control.

Lipid peroxidation studies were performed on fresh sera and synovial fluid in one patient only. Quantitative infrared thermal imaging could not be performed on this patient whose details are given below as a case history.

\section{CASE HISTORY}

The patient is a 40 -year-old Caucasian female who developed monoarthritis at age 9 . By 12 years of age

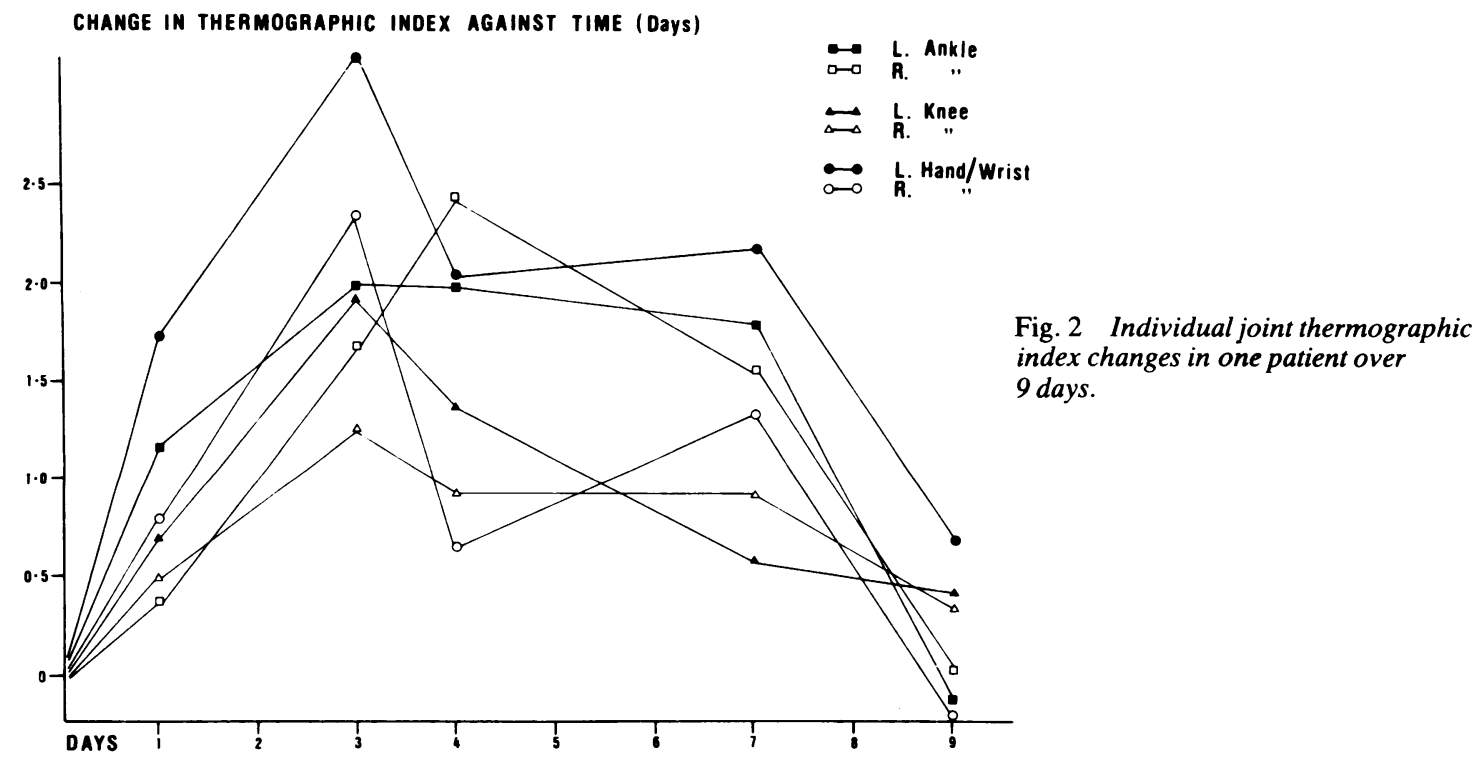

Fig. 2 Individual joint thermographic index changes in one patient over 9 days. 


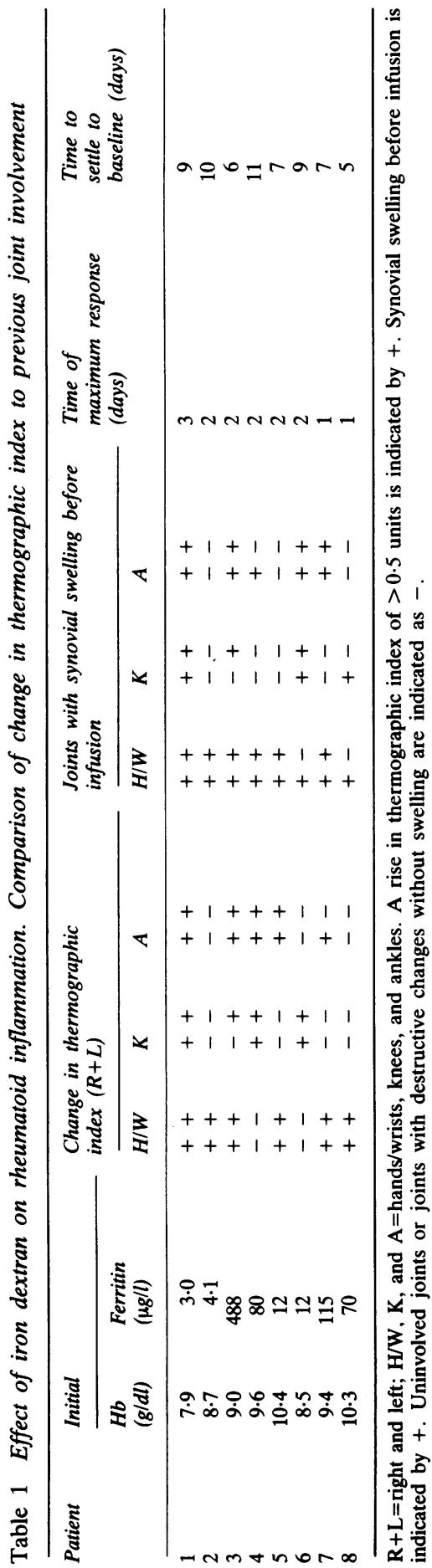

she had multiple joint involvement, fevers associ-믐. ated with exacerbations, but no rash, splenomegaly, or ocular problem. At the age of 20 the frequency of her 'flares' increased, and her salicylate maintenance therapy was changed to predisolone $(5 \mathrm{mg})$, 듬 on which dose she remained.

On three occasions she was prescribed oral雨 ferrous sulphate ( $200 \mathrm{mg}$ three times a day) for a lowo grade anaemia $(\mathrm{Hb} 11 \mathrm{~g} / \mathrm{dl})$, with indices suggestive ${ }^{\infty}$ of iron deficiency secondary to longstanding in -0 flammation (erythrocyte sedimentation rate $30-80-$ $\mathrm{mm} / \mathrm{h}$ ). Estimation of serum iron, iron binding capacity, or ferritin was not or could not bee performed. On each occasion the patient noted a mild exacerbation of her disease with a pyrexia (not quantified) and discontinued the treatment. At the ${ }^{\omega}$ age of 40 her $\mathrm{Hb}$ had dropped slowly to $7 \mathrm{~g} / \mathrm{dl}$, with aoo low serum iron, normal iron binding capacity, and serum ferritin of $20 \mu \mathrm{g} / \mathrm{l}$ (suggestive of iron deficiency in the presence of an ESR of $70 \mathrm{~mm} / \mathrm{h}$ ) ${ }^{14}{ }^{15}$ A sternal bone marrow revealed micronormoblastion erythropoiesis with reduced iron in the reticuloen- $\frac{\widehat{S}}{-}$ dothelial cells and none in erythroblasts. No specific cause of iron deficiency was found. The patientoo refused oral iron but gave informed consent for $a^{r}$ trial of intravenous iron dextran. This was infused ato a dose of $800 \mathrm{mg}$ over 8 hours, with hydrocortisone cover (100 mg IV) and her maintenance predniso-o lone doubled. Twelve hours after the infusion waso discontinued and despite strict bed rest for four days $\cong$ she developed a florid synovial reaction, with $\overrightarrow{\overrightarrow{0}}$ effusions, affecting the 2nd-4th metacarpopha-3 langeal joints, shoulders, hands, and ankles, all of? which had been previously damaged by ongoing? rheumatoid disease. Forty-eight hours after theor infusion she was pyrexial $\left(39^{\circ} \mathrm{C}\right.$ - oral) for 12 hours 0 (spikes of fever had been noted previously in association with episodes of synovitis). The synovia reaction slowly settled over three weeks, but did not? return to the preinfusion state, with morning stiff- ness increasing from $30 \mathrm{~min}$ to 2 hours and hers erythrocyte sedimentation rate consistently $>30 \mathrm{P}$ $\mathrm{mm} / \mathrm{h}$ more than baseline $(70 \mathrm{~mm} / \mathrm{h})$. Serum was tested for DCs and TBA reactive material directly after removal, prior to the infusion, and then at daily intervals for 4 days. Synovial fluid on removai was tested for TBA reactivity prior to the infusion w $^{2}$ (left knee) and then at the height of the reaction? (day 2 - left knee) (insufficient material was avail able for DC measurements). Table 2 gives details of these results.

Both TBA and DC levels rose in the sera after administration of iron dextran; peaking was concomitant with the flare. Unlike DC, TBA reactivity was surprisingly maintained at high levels for up to̊ 72 hours, which may suggest the presence of stable 
Table 2 The effect of $I V$ administration of iron dextran in the production of lipid peroxides in sera and synovial fluid

\begin{tabular}{|c|c|c|c|}
\hline \multirow[t]{2}{*}{ Day } & \multicolumn{2}{|l|}{ Serum } & \multirow{2}{*}{$\begin{array}{l}\text { Synovial fluid } \\
T B A \\
\text { (UV abs. } 532 \mathrm{~nm})\end{array}$} \\
\hline & $\begin{array}{l}D C \\
(U V \text { abs. } 240 \mathrm{~nm})\end{array}$ & $\begin{array}{l}T B A \\
(U V \text { abs. } 532 \mathrm{~nm})\end{array}$ & \\
\hline 0 & $0 \cdot 18$ & $0 \cdot 10$ & 0.00 \\
\hline 1 & 0.25 & $0 \cdot 18$ & - \\
\hline 2 & $0 \cdot 38$ & $0 \cdot 16$ & 0.58 \\
\hline 3 & $0 \cdot 14$ & $0 \cdot 13$ & - \\
\hline 4 & $0 \cdot 17$ & 0.08 & - \\
\hline
\end{tabular}

Sera and synovial fluid were collected immediately prior to the IV infusion of $800 \mathrm{mg}$ iron dextran (day 0 ). Sera and (synovial fluid $x$ 1) were then assessed daily.

The results are expressed as the mean of duplicate analyses made on fresh samples, and analysed as detailed in Methods.

Table 3 The potential of iron dextran (Imferon) in vitro to decompose lipid peroxides and to stimulate further peroxidation in normal sera

TBA (UV

abs. $532 \mathrm{~nm})$

Normal serum

0.01

Normal serum + dextran (mol.wt 90000 ) 0.01

Normal serum $+25 \mu \mathrm{M} \mathrm{Fe} \mathrm{Fe}^{2+}$ (iron dextran) 0.52

Normal serum $+250 \mu \mathrm{M} \mathrm{Fe}^{2+}$ (iron dextran) 0.85

Results are expressed as the mean of duplicate analyses. Iron concentrations are expressed as final concentrations in the reaction systems, which were analysed as detailed in 'Methods'.

aldehyde products. No TBA reactive material was present in the preinfusion synovial fluid. However, at day 2 levels of TBA reactivity were elevated and almost four times higher than the paired serum.

An in-vitro study was subsequently performed to assess the potential of iron dextran and dextran alone to stimulate lipid peroxidation in normal serum. Table 3 gives details of the results and methodology, and indicates that dextran alone has no effect on lipid peroxidation whereas iron dextran is considerably stimulatory.

\section{Discussion}

Intravenous iron dextran is prescribed for the correction of iron deficiency states when oral iron salt supplementation is considered inappropriate. However, its use in treating the 'anaemia of rheumatoid disease' is controversial. The diagnosis of genuine iron deficiency, as opposed to apparent iron deficiency due to chronic inflammation, is not evident from measurement of haemoglobin or red cell indices. A serum ferritin estimation is more useful despite an elevation at the lower limit of normality $(50-60 \mu \mathrm{g} / \mathrm{l})$ owing to the 'acute phase' nature of this protein. ${ }^{14} 15$ Four of the patients referred to us for study had ferritin values (retrospectively) above this range and were almost certainly not iron deficient; their anaemia would perhaps be better corrected by control of the inflammatory response.

Intravenous iron dextran is potentially a dangerous treatment, for death due to anaphylaxis has been reported. ${ }^{16}$ The onset and timing of the anaphylactic reaction resulting from iron dextran is unpredictable as shown in this study. Dextrans are known to cause allergic reactions of an 'immediate type' in a variable proportion of patients treated with them. The source of dextrans and techniques used in their manufacture appear to affect the incidence of this reaction, though antibodies may also develop naturally in response to dextrans present in foodstuffs or synthesised from strains of bacteria common in man. ${ }^{17}$ 'Skin' testing has been used to detect hypersensitivity to dextran, though it is unreliable as an index and potentially dangerous as a sensitiser. It was not performed in this study, but was uniformly negative in a previous report of iron dextran synovitis. ${ }^{1}$ Of the many and varied reactions to dextrans arthralgias are widely reported, but a clear inflammatory joint response has not been reported.

All our patients developed a synovial flare to iron dextran treatment which was clinically obvious in five out of eight. The striking feature of this phenomenon was that the inflammatory response was greatest in previously involved joints, and usually in those with existing clinically overt inflammation. This observation is consistent with that of Reddy and Lewis ${ }^{2}$ but contradictory to that inferred by Lloyd and Williams. ${ }^{1}$ The synovial flare in such a high proportion of patients might argue against a hypersensitivity reaction ${ }^{6}$ and suggests a direct relationship between a component of iron dextran and the inflammatory process. Our results tend to discount the view that a reticuloendothelial cell dysfunction and an immune complex synovitis are occurring ${ }^{6}$ and support the likelihood that the iron component of the dextran complex stimulates lipid peroxidation, which can augment inflammation.

Iron dextran is cleared from the circulation by the reticuloendothelial system and appears inside macrophages of the liver and spleen within an hour of injection. In healthy subjects the mean clearance half life is approximately 6 hours with $40 \%$ of the injected dose bound to transferrin 11 hours later. ${ }^{18}$ Only a small fraction of the complex bypasses this route and is not bound to transferrin. During inflammation the synovial membrane acts as an 
extension of the RE system, supporting a macrophage cell population capable of synthesising ferritin and storing iron. ${ }^{5}$ Gutteridge et al. have detected in synovial fluid a form of non-haem iron which is not bound to iron storage or iron transport proteins, such as transferrin and lactoferrin, but is capable of stimulating lipid peroxidation, ${ }^{19}$ a process known to occur in active synovitis. ${ }^{5}$ This "catalytic iron' is not detected in the serum of rheumatoid patients but can be removed in vitro from their synovial fluid by the addition of apotransferrin. ${ }^{20}$ Since the transferrin present in synovial fluid of patients with RA is not fully saturated with iron, ${ }^{20}$ it can be inferred that this protein has been damaged in some way within the inflamed joint. The 'acute phase' protein caeruloplasmin, which catalyses oxidation of ferrous ions to the ferric state and thereby aids incorporation of ferric iron into transferrin, is known to be damaged within inflamed joints, displaying a low catalytic activity towards iron oxidation compared with the total amount of immunologically reactive protein present. ${ }^{21}$

The following reaction sequence, indicated by our results, would explain the clinical phenomenon of 'joint flare'. Iron dextran is cleared by the reticuloendothelial system and the synovial macrophages, a process which results in the liberation of iron salts from the complex. Usually, in the absence of increased levels of lipid peroxides and with transferrin functioning normally, iron is transferred to the bone marrow for normal erythropoiesis. However, during synovial inflammation damage appears to occur to both the iron-transporting protein transferrin and the iron oxidation catalyst caeruloplasmin (ferroxidase). Such impairment would have serious implications for antioxidant protection within the synovial joint. ${ }^{22}{ }^{23}$ Under these circumstances iron complexes would be particularly toxic as both initiators and stimulators of further damage to lipids, proteins, and hyaluronic acid. An exacerbation, by a process of amplification, of existing inflammatory joint disease would then be expected following release of proteolytic enzymes and chemotactic factors. ${ }^{5} 13$

DRB and JL are supported by a research grant from the West Midlands Regional Health Authority, and Ciba-Geigy Pharmaceuticals, Horsham. We are grateful to the physicians of the Royal National Hospital for Rheumatic Diseases, Bath, who allowed us to study their patients, and to Fisons UK (Loughborough) for assisting with the literature search.

\section{References}

1 Lloyd K N, Williams P. Reactions to total dose infusion of iron dextran in rheumatoid arthritis. $\mathrm{Br}$ Med $J$ 1970; ii: 323-5.
2 Reddy $P$ S, Lewis $M$. The adverse effect of intravenouses: iron-dextran in rheumatoid arthritis. Arthritis Rheum 1969; 12: 454-7.

3 Jones C E M, Mowat A G. Total dose infusion of iron dextran in rheumatoid arthritis. Rheum Phys Med 1972; 11: 240-5.

4 Thaman O P, Dogra K N. Total dose parenteral iron therapy in children. Indian $J$ Paediatr 1968; 35: 1-5.

5 Blake D R, Hall N D, Bacon P A, Dieppe P A, Halliwell B $\overparen{D}$ Gutteridge J M C. The importance of iron in rheumatoic disease. Lancet 1981; ii: 1142-4.

6 Ron D. The reticuloendothelial system and rheumatoid arthri- $\overrightarrow{0}$ tis. Lancet 1979; ii: 901.

7 Ropes M W, Bennett G A, Cobb S, Jacox R, Jessar R A. 1958 revision of diagnostic criteria for rheumatoid arthritis. Bullor Rheum Dis 1958; 9: 175-6.

8 Collins A J, Ring E F J, Cosh J A, Bacon P A. Quantitation of thermography in arthritis using multi-isothermal analysis 1 . The thermographic index. Ann Rheum Dis 1974; 33: 113-5.

9 Blake D R, Bacon P A, Joyce H J, Scott D G I, Wallington T B. The scope of immune complexes in clinical rheumatology. In: $\infty$ Vello G P, ed. Rheumatoid arthritis. New York: Raven Press $\omega$ 1981: 81-90. (Advances in inflammation research; vol 2).

10 Bradfield J W B. A new look at reticuloendothelial blockade. Br J Exp Pathol 1980; 61: 617-23.

11 Satoh K. Serum lipid peroxides in cerebrovascular disorders determined by a new colorimetric method. Clin Chim Acta $\stackrel{\rho}{S}$ 1978; 90: $37-43$.

12 Lunec J, Halloran S P, White A G, Dormandy T L. Free radica oxidation (peroxidation) products in serum and synovial incr rheumatoid arthritis. J Rheumatol 1981; 8: 233-45.

13 McBrien D C H, Slater T F, eds. Free radicals, lipido peroxidation and cancer. London: Academic Press, 1982.

14 Hansen T M, Hansen N E, Birgens H S, Holund B, Lorenzen I. $\overline{0}$ Serum ferritin and the assessment of iron deficiency in rheumatoid arthritis. Scand J Rheumatol 1983; 12: 353-9.

15 Blake D R, Waterworth R F, Bacon P A. The assessment of iron stores in inflammation by assay of serum ferritin concentra-⿳亠口冋 tions. Br Med J 1981; 283: 1147-8.

16 Zipf R E. Fatal anaphylaxis after intravenous iron dextran. Forensic Sci 1975; 20: 326-33.

17 Kabat E A, Turino G M, Tarrow A B, Mauver P H. Studies on the immunochemical basis of allergic reactions to dextran in man. J Clin Invest 1957; 36: 1160-70.

18 Kanak Korn K, Cavill I, Jacobs A. The metabolism of intravenously administered iron-dextran. Br J Haematol 1973 25: $637-43$

19 Gutteridge J M C, Rowley D A, Halliwell B. Superoxidedependent formation of hydroxyl radicals in the presence ofo iron salts. Detection of free iron in biological systems by the bleomycin-dependent degradation of DNA. Biochem J 1981; I 199: 263-5.

20 Gutteridge J M C, Halliwell B, Rowley D A. Catalytic iron complexes in biological material: a potential for oxygen radicalo damage. Life Chem Rep in press.

21 Blake D R, Blann A, Bacon P A, Gutteridge J M C, Farr M, N Halliwell B. Ferroxidase and ascorbate oxidase activities of $\omega$ caeruloplasmin in synovial fluid from rheumatoid patients. Clin Sci Mol Med 1983; 64: 551-3.

22 Gutteridge J M C, Stocks J. Caeruloplasmin: physiological and pathological perspectives. CRC Crit Rev Clin Lab Sci 1981; 14: ¿ 257-329.

23 Gutteridge J M C. Antioxidant properties of caeruloplasmin towards iron and copper-dependent oxygen radical formation. FEBS Lett 1983; 157: 37-40. 